\title{
The Influence of Glucose, Ammonium and Magnesium Availability on the Production of Protease and Bacitracin by Bacillus licheniformis
}

\author{
By G. W. HANLON, ${ }^{1}$ N. A. HODGES ${ }^{*}$ AND A. D. RUSSELL \\ ${ }^{1}$ Department of Pharmacy, Brighton Polytechnic, Moulsecoomb, Brighton, \\ Sussex BN2 4GJ, U.K. \\ ${ }^{2}$ Welsh School of Pharmacy, UWIST, Cardiff CF1 3NU, U.K.
}

(Received 10 June 1981; revised 11 August 1981)

\begin{abstract}
Bacillus licheniformis was cultivated in a range of defined media varying in both the nature of the growth-limiting component and the concentration of excess nutrients. The compositions of the media were such as to ensure that the final absorbance $\left(A_{430}\right)$ of the culture was the same in each case. Samples taken during the stationary phase were assayed for their content of extracellular serine protease and bacitracin. The nature of the growth-limiting nutrient had a profound effect on the amounts of these products formed while those components which were present in excess also exerted an influence in proportion to their concentration. Thus, for example, a four-fold increase in serine protease production occurred when ammonium replaced glucose as the growth-limiting nutrient. Serine protease and bacitracin production responded differently to these varying cultural conditions suggesting they are subject to separate control mechanisms. The results are discussed in relation to the need for rigorously controlled cultural conditions during physiological studies of this nature.
\end{abstract}

\section{INTRODUCTION}

Spore formation and the synthesis of extracellular protease and antibiotic are coincident processes which are promoted by conditions of nutrient exhaustion in batch cultures or restricted growth rates in continuous culture. Although it is now firmly established that antibiotic production is not an essential step in normal spore development, the requirement for serine protease is still uncertain (Young \& Mandelstam, 1980). The mechanisms controlling the three processes are similarly ill-defined, although catabolite repression systems have been suggested for each (Laishley \& Bernlohr, 1968; Martin \& Demain, 1980; Young \& Mandelstam, 1980). Using mutants of Bacillus subtilis, Schaeffer (1967) demonstrated that the properties of antibiotic production and protease production were frequently, but not invariably, associated. Sadoff and co-workers subsequently reported that antibiotic peptides resulted from the action of serine protease on vegetative cell material, the implication of this being that the concentration of these antibiotics was influenced by that of the protease (Vitković \& Sadoff, 1975, 1977).

The influence of culture medium composition and exhaustion of specific nutrients on the formation, quantity and physiological characteristics of spores is well established (Grelet, 1957; Dawes \& Mandelstam, 1970; Hodges \& Brown, 1975). Extracellular product formation, however, has often been examined under less well-defined conditions than those employed for the study of sporulation per se. Priest (1977) has criticized much of the work on extracellular enzyme synthesis for the use of poorly classified and misnamed organisms, culture media of variable and unknown composition and the development of individual assay procedures which hindered the comparison of results from different laboratories. Wouters \& Buysman (1977) have similarly commented on a lack of systematic study designed to elucidate those factors which control the synthesis and release of extracellular enzymes. 
The ease of utilization of various carbon and nitrogen sources has recently been shown markedly to influence the concentrations of protease and bacitracin achieved in batch cultures of Bacillus licheniformis (Hanlon \& Hodges, 1981b) and the importance of magnesium availability in sporulation of Bacillus species is long established (Grelet, 1957). In view of these facts and the comments of Priest (1977) and Wouters \& Buysman (1977) above, this present work was undertaken with the intention of developing a minimal medium suitable for growth and production of bacitracin, serine protease and spores by $B$. licheniformis, and investigating the influences of glucose, ammonium and magnesium availability on these processes under conditions which preclude effects due to changes in growth rate or final cell density.

\section{METHODS}

Organism, cultural conditions and media. Bacillus licheniformis NCIB 8874 was grown at $37{ }^{\circ} \mathrm{C}$ in $250 \mathrm{ml}$ conical flasks containing $50 \mathrm{ml}$ medium on a reciprocating shaker operating at $100 \times 6 \mathrm{~cm}$ throws per min. Growth was recorded as absorbance at $430 \mathrm{~nm}\left(A_{430}\right)$ in a Unicam SP500 spectrophotometer. The medium contained (mM): glucose, $15 ; \mathrm{Na}_{2} \mathrm{HPO}_{4}, 40 ; \mathrm{KH}_{2} \mathrm{PO}_{4}, 26 ; \mathrm{NH}_{4} \mathrm{Cl}, 10 ; \mathrm{MgSO}_{4}, 0.5 ; \mathrm{MnCl}_{2}, 0 \cdot 01 ; \mathrm{FeSO}_{4}, 0.01$; $\mathrm{CaCl}_{2}, 0.1 ; \mathrm{pH} \mathrm{7.0.} \mathrm{Any} \mathrm{alterations} \mathrm{to} \mathrm{these} \mathrm{concentrations} \mathrm{are} \mathrm{noted} \mathrm{in} \mathrm{the} \mathrm{text.} \mathrm{Cultures} \mathrm{received} \mathrm{an}$ exponential-phase vegetative cell inoculum growing in a medium of identical composition.

Chemicals. All chemicals used in the media were of analytical grade. Bacitracin, serine protease and phenylmethylsulphonyl fluoride (PMSF) were obtained from Sigma.

Bacitracin assay. Samples were removed from the cultures at the times indicated, immediately chilled and centrifuged at $4{ }^{\circ} \mathrm{C}$ for $15 \mathrm{~min}$ at $10000 \mathrm{~g}$. The supernatants were subjected to a cup-plate agar-diffusion assay in Diagnostic Sensitivity Test agar (Oxoid) using Micrococcus luteus as the test organism. Concentrations were expressed in international units $\mathrm{ml}^{-1}$.

Protease assay. Culture supernatant $(1 \mathrm{ml})$ was placed in a tube together with $3 \mathrm{ml} \mathrm{pH} 7.0$ phosphate buffer and equilibrated to $37^{\circ} \mathrm{C}$. Then $2 \mathrm{ml}$ pre-warmed azo-albumin solution $\left(12.5 \mathrm{mg} \mathrm{ml}^{-1}\right)$ was added, mixed thoroughly and incubated for $30 \mathrm{~min}$. The reaction was stopped by the addition of $4 \mathrm{ml} 10 \%(\mathrm{w} / \mathrm{v})$ trichloroacetic acid and the resultant precipitate was filtered through a Whatman no. 1 paper. To $5 \mathrm{ml}$ of the filtrate was added $5 \mathrm{ml}$ $0.5 \mathrm{M}-\mathrm{NaOH}$ immediately prior to reading the absorbance at $440 \mathrm{~nm}$. Samples were also assayed in the presence of PMSF (1 mM) which abolished approximately $98 \%$ of the total activity. The activity in the supernatant was therefore considered to be due to alkaline serine protease rather than neutral metallo-protease. Units of activity were those defined by Sigma for crystalline serine protease which was used as a reference standard.

Sporulation. The percentage of refractile spores was determined directly by counting the number of free spores and sporangia using a phase-contrast microscope.

\section{RESULTS}

Media were developed in which either glucose or ammonium chloride was present at a growth-limiting concentration $(4.4 \mathrm{mM}$ and $2.3 \mathrm{mM}$, respectively) sufficient only to support growth to an $A_{430}$ of 1.5. All other medium components were present in excess sufficient to support growth to an $A_{430}$ of 5.0. Preliminary experiments using these media showed that protease concentrations normally reached a plateau after approximately $40 \mathrm{~h}$ incubation, whereas the concentration of bacitracin reached a peak after about $30 \mathrm{~h}$ with a slow decline thereafter.

Protease and bacitracin concentrations were recorded in cultures grown in a glucoseexhausted medium with a range of ammonium chloride concentrations (Fig. 1). Turbidity measurements and microscopical examination indicated no differences in the rate of growth or appearance of spores in these cultures. Both bacitracin and protease began to appear 16-18 $\mathrm{h}$ after inoculation and the same pattern of production was observed in each case with the levels of activity depending upon the concentration of ammonium chloride in the medium. At high concentrations the production of both bacitracin and protease was repressed and this repression decreased as the concentration of nitrogen was reduced. The possibility exists that this is a non-specific ionic effect but preliminary experiments using similar media with 

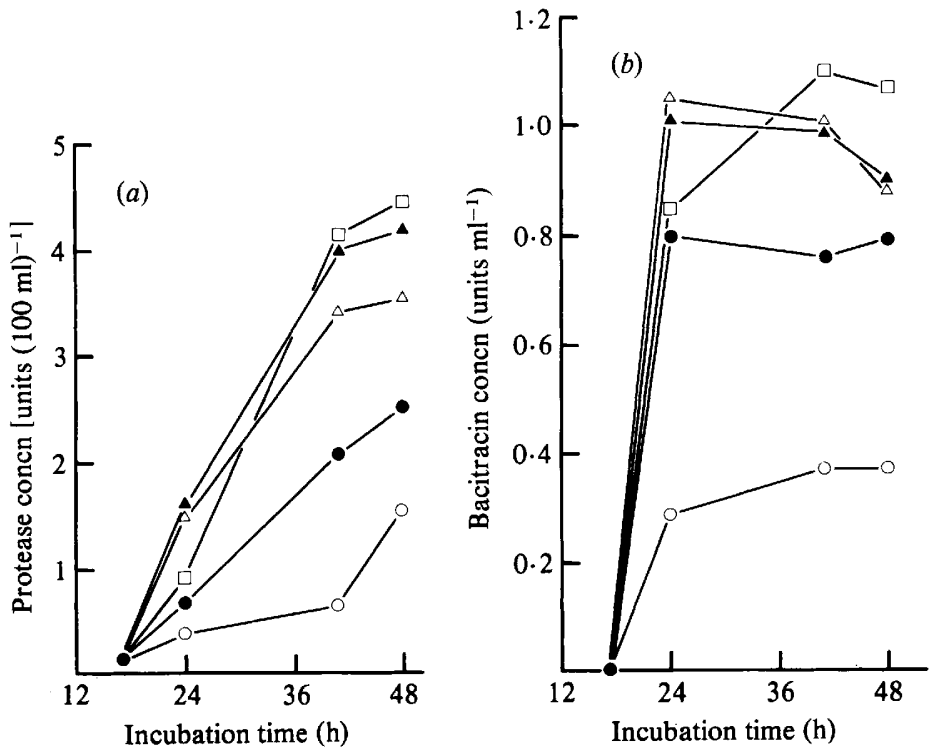

Fig. 1. Production of protease $(a)$ and bacitracin $(b)$ by glucose-exhausted cultures showing the effect of excess ammonium. Concentrations of ammonium chloride were $300 \mathrm{~mm}(\mathrm{O}), 150 \mathrm{~mm}(\mathrm{O}), 50 \mathrm{~mm}$ $(\triangle), 15 \mathrm{~mm}(\Delta)$ and $3 \mathrm{~mm}(\square)$.
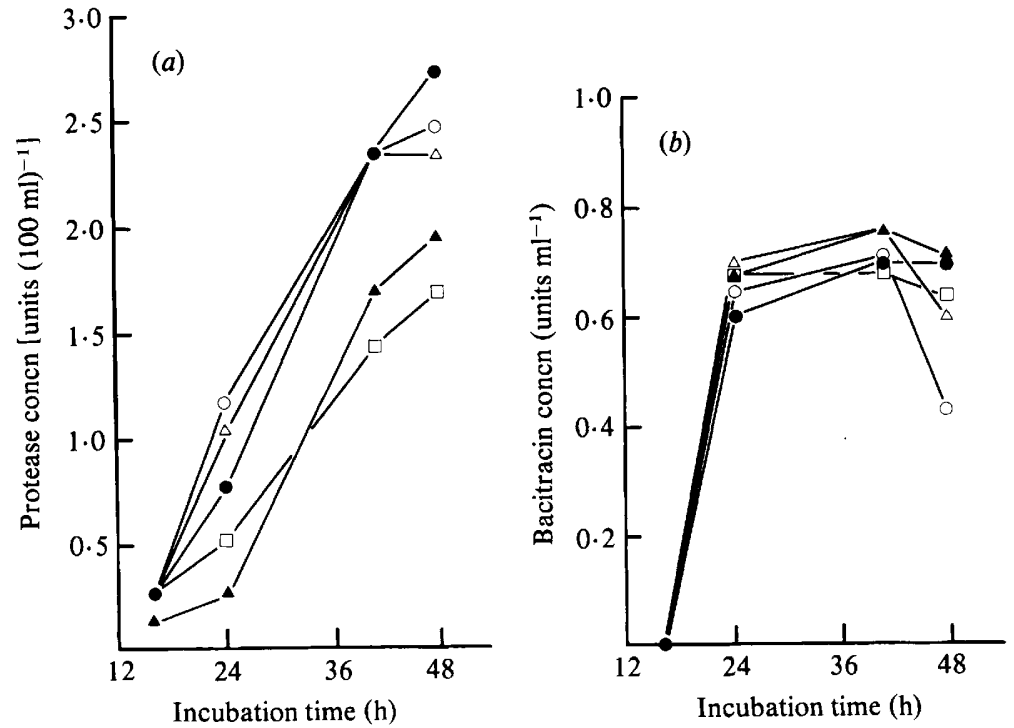

Fig. 2. Production of protease $(a)$ and bacitracin $(b)$ by glucose-exhausted cultures showing the effect

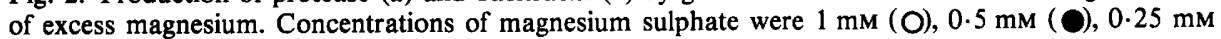
$(\triangle), 0.1 \mathrm{mM}(\triangle)$ and $0.05 \mathrm{mM}(\square)$.

increased phosphate concentrations gave no evidence to support this (data not shown), and work by other authors suggests that it is likely to be an ammonium-specific repression (Levisohn \& Aronson, 1967; Martin \& Demain, 1980).

A second glucose-exhausted medium was prepared with a constant concentration of excess ammonium chloride (10 mM) and a range of magnesium concentrations (Fig. 2), the lowest of 

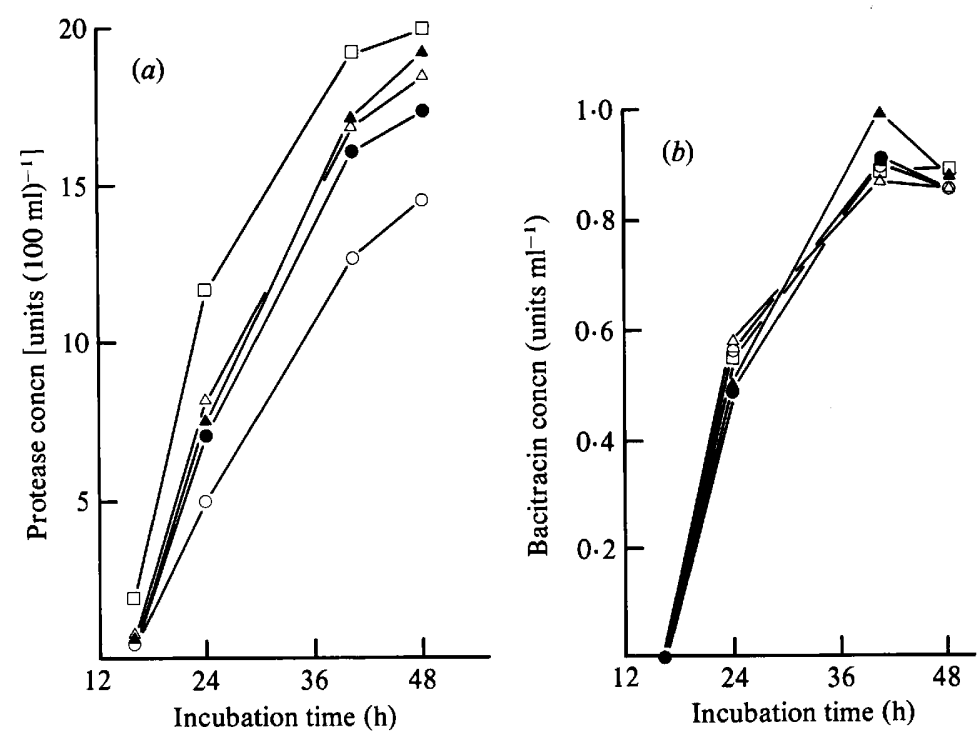

Fig. 3. Production of protease $(a)$ and bacitracin $(b)$ by nitrogen-exhausted cultures showing the effect of excess glucose. Concentrations of glucose were $250 \mathrm{~mm}(\mathrm{O}), 150 \mathrm{mM}(\bigcirc), 100 \mathrm{mM}(\triangle), 50 \mathrm{mM}(\Delta)$ and $15 \mathrm{mM}(\square)$.
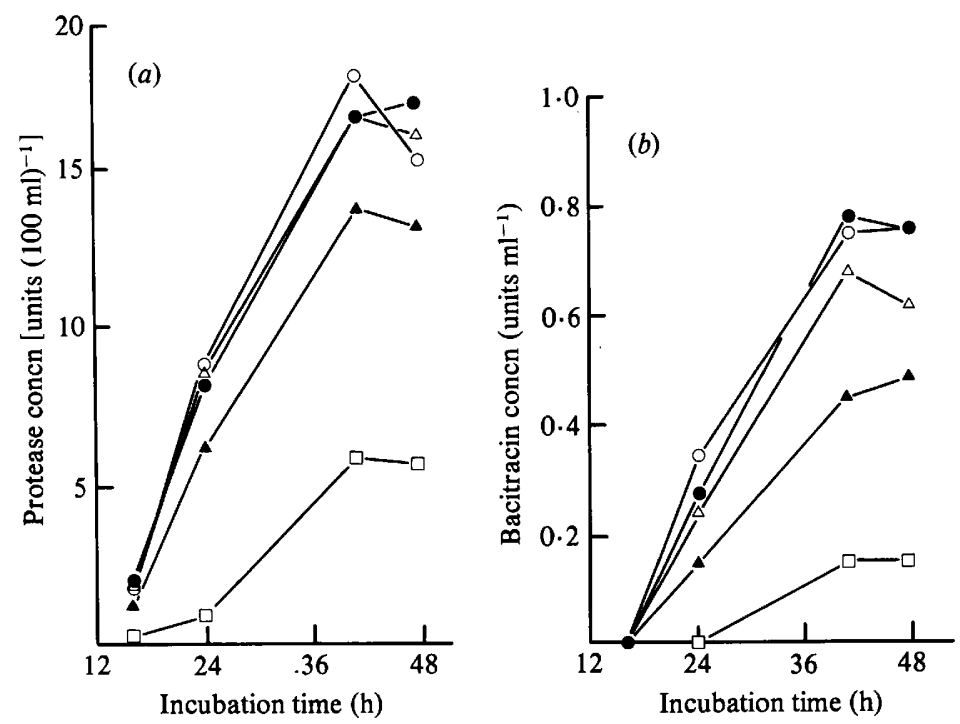

Fig. 4. Production of protease $(a)$ and bacitracin $(b)$ by nitrogen-exhausted cultures showing the effect of excess magnesium. Concentrations of magnesium sulphate were $1 \mathrm{mM}(O), 0.5 \mathrm{mM}(\bigcirc), 0.25 \mathrm{~mm}$ $(\triangle), 0.1 \mathrm{mM}(\Delta)$ and $0.05 \mathrm{mM}(\square)$.

which was just sufficient to prevent the medium from becoming magnesium-limited. The results showed the protease concentration to be independent of magnesium availability at concentrations of $0.25 \mathrm{mM}^{-} \mathrm{MgSO}_{4}$ and above; at lower concentrations protease production was diminished. Bacitracin concentrations were unaffected by the changes in magnesium concentration. 


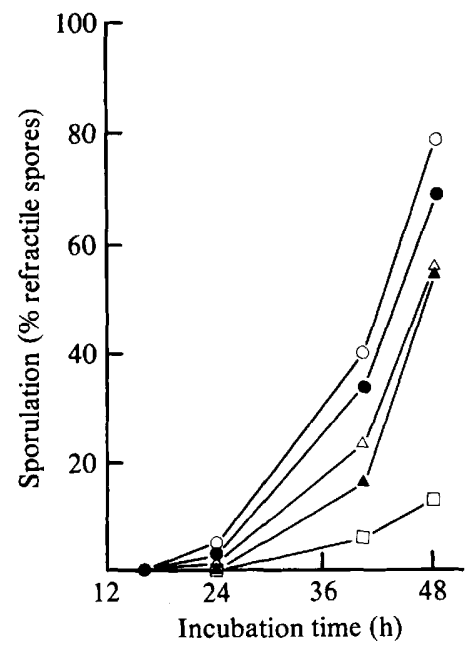

Fig. 5. Effect of magnesium availability on the sporulation of nitrogen-exhausted cultures. Magnesium sulphate concentrations were as in Fig. 4.

A range of nitrogen-exhausted media were prepared in which either glucose or magnesium were present in different concentrations. The glucose concentrations employed in the cultures (Fig. 3) were sufficient not only to support growth to the required cell density but also to satisfy energy requirements for metabolism throughout the experimental period after growth had ceased. The results show the high concentrations of protease characteristic of nitrogen-exhausted cultures and also illustrate a repressing effect on the production of protease by increasing concentrations of glucose (Fig. $3 a$ ). This repressing effect was not seen with bacitracin (Fig. $3 b$ ). Unlike the situation in glucose-exhausted media, a microscopical analysis of the cultures revealed a marked difference in the rate of appearance of refractile spores when different magnesium concentrations were employed in nitrogenexhausted media (Fig. 5). Although the rate of sporulation decreased as the magnesium concentration was reduced, the production of protease and bacitracin were little affected at concentrations in excess of $0.25 \mathrm{mM}^{-\mathrm{MgSO}_{4}}$. At lower concentrations, however, the rate of formation of both extracellular products was markedly reduced (Fig. 4).

\section{DISCUSSION}

Our results show that the patterns of synthesis of both protease and bacitracin are markedly influenced not only by the nature of the nutrient that, when exhausted, limits vegetative growth, but also by the degree of excess of non-limiting nutrients. Certain of the results further indicate that protease synthesis and bacitracin synthesis are subject to different control mechanisms.

Protease concentrations in ammonium-exhausted cultures were consistently higher than those in glucose-exhausted cultures, whereas the bacitracin concentrations were similar. The difference in protease concentrations is due primarily to a requirement for glucose as an energy source during the period of protease synthesis: in cultures simultaneously depleted of both glucose and nitrogen the protease concentrations were those characteristic of glucose depletion alone (Hanlon \& Hodges, 1981a, b). A similar effect of carbon and nitrogen limitation on protease production by chemostat cultures of $B$. licheniformis had previously been reported by Wouters \& Buysman (1977) but the protease in question was not specified.

The nature of the growth-limiting nutrient also influenced the rate of spore formation and 
the time of the peak concentrations of bacitracin but not of protease. Although the final percentage of spores was similar in each case, spore development in glucose-exhausted cultures preceded that in nitrogen-exhausted cultures by approximately $6 \mathrm{~h}$. The peak bacitracin concentrations were similarly earlier in glucose-exhausted cultures but protease was unaffected (data not shown). Repression of protease by high concentrations of excess ammonium chloride (Fig. $1 a$ ) and glucose (Fig. $3 a$ ) occurred, and in this respect the results reported here are in agreement with those of Levisohn \& Aronson (1967) and Laishley \& Bernlohr (1968), respectively.

Martin \& Demain (1980) have reviewed the few reports of nitrogen metabolite control of antibiotic synthesis and have indicated the possibility that the presence of ammonium may depress production. Consequently, the reduction in bacitracin concentration observed to result from excess ammonium (Fig. $1 b$ ) was not unexpected. The apparent absence of any repressing effect of glucose on bacitracin (Fig. $3 b$ ) was surprising in view of the numerous reports of regulation by glucose of antibiotic synthesis in general and that of bacitracin in particular (Haavik, 1974a, b). Much of this work, however, has been conducted under conditions in which the addition of glucose may have resulted in an increase in the growth rate of the culture. Martin \& Demain (1980) have suggested that the molecular mechanism of carbon catabolite regulation of antibiotic synthesis may be related to growth rate control. If this were so, the repression by glucose may be more an effect due to a growth rate change than one due to the presence of glucose per se. The absence of nitrogen to support further growth precluded the possibility of growth rate effects influencing the results described in Fig. $3(b)$.

Our results are interesting also in that the bacitracin and protease concentrations are not similarly affected by glucose excess. This suggests that bacitracin and protease are subject to different control mechanisms, and that the former is not merely a consequence of the latter as indicated by Vitkovic \& Sadoff (1977). The fact that the characteristically high protease concentrations achieved in nitrogen-exhausted cultures are not reflected by higher concentrations of bacitracin in nitrogen- than glucose-exhausted cultures further supports this contention.

There was a complete absence of sporulation in cultures grown in magnesium-exhausted medium and the concentrations of protease and bacitracin were negligible (data not shown). When present in excess, however, the concentration of magnesium had a profound effect on the concentrations of protease and bacitracin in glucose- and nitrogen-exhausted cultures. When the magnesium concentration was reduced from 0.25 to $0.1 \mathrm{mM}$ or less, a reduction in protease concentration occurred in both glucose- and nitrogen-exhausted cultures (Figs $2 a$, $4 a$ ). In the latter, bacitracin was similarly affected, but the antibiotic concentration was unaffected by the magnesium concentration in glucose-exhausted cultures: this is a further indication that protease and bacitracin synthesis are subject to independent control.

The effects of magnesium availability are due, presumably, to the requirement for this cation in protein synthesis, its depletion restricting enzyme synthesis. Frøyshov (1974) has reported that bacitracin is manufactured by a three-component enzyme complex. The failure of reduced magnesium availability (Fig. $2 b$ ) to influence bacitracin production suggests sufficient of this enzyme complex was present for antibiotic synthesis to proceed normally. The appearance of bacitracin in glucose-exhausted cultures earlier than in nitrogen-exhausted cultures suggests that the synthetases may be produced in glucose-exhausted cultures before the rate of protein synthesis is affected by magnesium availability. Sporulation, also, was influenced by magnesium concentration only in nitrogen-exhausted cultures (Fig. 5), the rate of spore formation in glucose-exhausted cultures being unaffected (data not shown).

These results therefore indicate the importance of precisely defining the experimental conditions under which studies on the relationships between sporulation, protease and antibiotic production are undertaken. Media defined not only in terms of the proportions of purified ingredients but also in terms of nutrient exhaustion or relative excess are clearly 
desirable to eliminate misleading effects due to changes in cell density and thus, possibly, oxygen availability per cell, and to distinguish effects resulting from growth rate changes from those due to the presence or absence of specific chemicals themselves.

\section{REFERENCES}

Dawes, I. W. \& Mandelstam, J. (1970). Sporulation of Bacillus subtilis in continuous culture. Journal of Bacteriology 103, 529-535.

FrøYsHov, Ø. (1974). Bacitracin biosynthesis by three complementary fractions from Bacillus licheniformis. FEBS Letters 44, 75-78.

GRELET, N. (1957). Growth limitation and sporulation. Journal of Applied Bacteriology 20, 315-324.

HAAVIK, H. I. (1974a). Studies on the formation of bacitracin by Bacillus licheniformis: effect of glucose. Journal of General Microbiology 81, 383-390.

HAAvIK, H. I. (1974 b). Studies on the formation of bacitracin by Bacillus licheniformis: role of catabolite repression and organic acids. Journal of General Microbiology 84, 321-326.

Hanlon, G. W. \& Hodges, N. A. (1981a). Requirement for glucose during production of extracellular serine protease by cultures of Bacillus licheniformis. FEMS Microbiology Letters 11, 51-54.

HANLON, G. W. \& Hodges, N. A. (1981b). Bacitracin and protease production in relation to sporulation during exponential growth of Bacillus licheniformis on poorly utilized carbon and nitrogen sources. Journal of Bacteriology 147, 427-431.

Hodges, N. A. \& Brown, M. R. W. (1975). Properties of Bacillus megaterium spores formed under conditions of nutrient limitation. In Spores VI, pp. 550-555. Edited by P. Gerhardt, R. N. Costilow \& H. L. Sadoff. Washington, D.C.: American Society of Microbiology.

LAISHLEY, E. J. \& BERNLOHR, R. W. (1968). Regula- tion of arginine, and proline catabolism in Bacillus licheniformis. Journal of Bacteriology 96, 322329.

LEvisohn, S. \& ARonson, A. I. (1967). Regulation of extracellular protease production in Bacillus cereus. Journal of Bacteriology 93, 1023-1030.

MaRTin, J. F. \& DEMAin, A. L. (1980). Control of antibiotic biosynthesis. Microbiological Reviews 44, 230-251.

PrIEST, F. G. (1977). Extracellular enzyme synthesis in the genus Bacillus. Bacteriological Reviews 41, 711-753.

SCHAEFFER, P. (1967). Asporogeneous mutants of Bacillus subtilis Marburg. Folia microbiologica 12 , 291-296.

VITKović, L. \& SADOFF, H. L. (1975). Relationship between sporulation, protease and antibiotic in sporulating Bacillus licheniformis cells. In Spores $V I$, pp. 362-366. Edited by P. Gerhardt, R. N. Costilow \& H. L. Sadoff. Washington, D.C.: American Society for Microbiology.

VITković, L. \& SADOFF, H. L. (1977). In vitro production of bacitracin by proteolysis of vegetative Bacillus licheniformis cell protein. Journal of Bacteriology 131, 897-905.

Wouters, J. T. M. \& BuYsman, P. J. (1977). Production of some extracellular enzymes by Bacillus licheniformis $749 / \mathrm{C}$ in continuous cultures. FEMS Microbiology Letters 1, 109-112.

Young, M. \& MANDELSTAM, J. (1980). Early events during bacterial endospore formation. Advances in Microbial Physiology 20, 103-162. 